Comment. Math. Helv. 72 (1997) 389-399

(C) 1997 Birkhäuser Verlag, Basel

$0010-2571 / 97 / 030389-11 \$ 1.50+0.20 / 0$

Commentarii Mathematici Helvetici

\title{
Picard groups of multiplicative invariants
}

\author{
Martin Lorenz*
}

\begin{abstract}
Let $S=k A$ denote the group algebra of a finitely generated free abelian group $A$ over the field $k$ and let $G$ be a finite subgroup of $\operatorname{GL}(A)$. Then $G$ acts on $S$ by means of the unique extension of the natural $\mathrm{GL}(A)$-action on $A$. We determine the Picard group Pic $R$ of the algebra of invariants $R=S^{G}$. As an application, we produce new polycyclic group algebras with nontrivial torsion in $K_{0}$.
\end{abstract}

Mathematics Subject Classification (1991). Primary: 13A50, 13C20, 13D15, 16E20, 16W20, 19A31; Secondary: 16S34, 20H15

Keywords. Picard group, ring of invariants, group action, Grothendieck group, polycyclic group algebra, Cartan map, class group, 1-cohomology group

\section{Introduction}

It appears to be unknown whether all finitely generated projective modules over rings of invariants for rational linear actions of reductive algebraic groups on affine space $\mathbb{A}^{n}$ over an algebraically closed field of characteristic 0 are stably free, or even free (cf. $[\mathrm{Kr}]$, Problem 5.1). For finite groups, stable freeness has recently been established by M. Holland [Ho] in the special case where the underlying representation decomposes into fixed point free and trivial constituents only. Our goal here is to show that, for multiplicative actions of finite groups, the analog of Kraft's question has a negative answer. In fact, we determine the Picard group Pic $R$ of multiplicative invariant rings $R$ and exhibit examples where it is nontrivial. As is well-known $[\mathrm{K}]$ (or see $\S 2.3$ below), the latter cannot happen for linear actions.

Specifically, let $S=k A$ denote the group algebra of a finitely generated free abelian group $A$ over the field $k$ and let $G$ be a finite subgroup of $\operatorname{GL}(A)$, acting on $S$ by means of the $k$-linear extension of the natural action on $A$. Working under the assumption that the order of $G$ is nonzero in $k$, we show that the Picard

* Research supported in part by NSF Grant DMS-9400643. 
group Pic $R$ of the invariant algebra $R=S^{G}$ measures the extent to which the 1-cohomology $\mathrm{H}^{1}(G, A)$ fails to be detected by the cyclic subgroups of $G$, to wit:

Theorem. Pic $R \cong \bigcap_{C} \operatorname{Ker}\left(\operatorname{Res}_{C}^{G}: \mathrm{H}^{1}(G, A) \rightarrow \mathrm{H}^{1}(C, A)\right)$, where $C$ runs over the cyclic subgroups of $G$.

A systematic search of the groups $G$ arising from lattices $A$ of rank at most 4 , conducted with the aid of the crystallographic groups library in $G A P$ [Sch], reveals that Pic $R$ is seldom nontrivial, but such cases do occur: 2 for rk $A=3$ (out of a total of 73 ), and 9 (out of 710) for $\operatorname{rk} A=4$.

Two noteworthy facts about $\operatorname{Pic} R$ are as follows. First, Pic $R$ survives the Cartan map $K_{0}(R) \rightarrow G_{0}(R)$ via its canonical embedding into the class group $\mathrm{Cl} R$ (see $\S 3.6$ ). Thus, our findings show that, other than in the case of linear invariants (see the Appendix), the image of the Cartan map for multiplicative invariants is not necessarily generated by the classes of free modules. Second, we show in $\S 3.7$ that Pic $R$ is always a subquotient of the torsion subgroup of $K_{0}(k \Gamma)$, where $\Gamma$ denotes the semidirect product $A \rtimes G$. Thus our examples give rise to new polycyclic-by-finite groups $\Gamma$ such that $K_{0}(k \Gamma)$ has nontrivial torsion, the only instance of this kind known hitherto being a construction due to Kropholler and Moselle $[\mathrm{KM}]$. The (multiplicative) invariant algebras $R$ coming from the Kropholler-Moselle groups actually have a trivial Picard group; so Pic $R$ is not solely responsible for torsion in $K_{0}(k \Gamma)$.

The first part of the article, Section 2, deals quite generally with actions of finite groups on arbitrary commutative rings $S$, assuming only that the every invariant is a trace. We give an account, without proofs, of the description of the Picard group of the invariant ring. Couched in geometric terms (and working over an algebraically closed base field of characteristic 0 ), all this can be found in $[\mathrm{Kr}]$ and $[\mathrm{DMV}]$, even for rational actions of reductive algebraic groups, and we have simply transfigured this material into a ring theoretic setting suitable for our purposes. Section 3 is then devoted entirely to multiplicative actions and contains the aforementioned results.

\section{Picard groups}

2.1. Throughout this section, $S$ will be a ring and $G$ will be a finite group acting by automorphisms on $S$, written $s \mapsto s^{g}$. Furthermore,

$$
\begin{array}{ll}
R=S^{G} & \text { will denote the ring of } G \text {-invariants in } S \text {, and } \\
T=S * G & \text { will be the skew group ring of } G \text { over } S .
\end{array}
$$

Thus $T$ is an associative ring containing $S$ as a subring, and $G$ is a subgroup of $\mathrm{U}(T)$, the group of units of $T$. The elements of $G$ form a free basis of $T$ as right $S$-module. Multiplication in $T$ is based on the rule $g a \cdot h b=g h a^{h} b$ for 
$a, b \in S, g, h \in G$. The ring $S$ becomes an $R$-T-bimodule via

$$
r \cdot a \cdot g b=r a^{g} b \quad(r \in R, a, b \in S, g \in G) .
$$

We will work under the standing hypothesis that the trace map $\operatorname{tr}: S \rightarrow R, s \mapsto$ $\sum_{g \in G} s^{g}$ is surjective or, equivalently:

$$
\text { There exists an element } x \in S \text { with } \operatorname{tr}(x)=1 \text {. }
$$

This entails in particular that $S_{T}$ is projective. Moreover, letting (. $)^{G}$ denote $G$-invariants, the functors

$$
\operatorname{proj}-R \underset{F}{\stackrel{E}{\longleftrightarrow}} \operatorname{add}\left(S_{T}\right), \quad E(Q)=Q \otimes_{R} S_{T}, \quad F(P)=P^{G}
$$

yield inverse equivalences of the category of finitely generated projective (right) $R$-modules proj- $R$ with the full subcategory $\operatorname{add}\left(S_{T}\right)$ of $\operatorname{proj}-T$ consisting of the direct summands of the modules $S_{T}^{n}$ for $n \geq 0$ (e.g., [Ho], §2.2).

\subsection{The $G$-Picard group of $S$}

For the remainder of this section, we assume that $S$ is commutative, and Max $S$ will denote the set of maximal ideals of $S$. For each $\mathfrak{M} \in \operatorname{Max} S$, we let $G^{T}(\mathfrak{M})=$ $\left\{g \in G: s^{g}-s \in \mathfrak{M}\right.$ for all $\left.s \in S\right\}$ denote the inertia group of $\mathfrak{M}$ and $(S / \mathfrak{M})^{*}$ the nonzero elements of $S / \mathfrak{M}$. Furthermore, we let

\section{$\operatorname{Pic}_{G} S$}

denote the set of isomorphism classes of those finitely generated $T$-modules $P$ that are invertible as $S$-modules, that is, $P_{\mathfrak{M}} \cong S_{\mathfrak{M}}$ holds for all $\mathfrak{M} \in \operatorname{Max} S$ (cf. [Ba], p. 132). These modules are projective over $S$, and hence over $T$ as well, by virtue of hypothesis $\left(^{*}\right)$ (e.g., $[\mathrm{H}]$, Proposition A.1). The tensor product $\otimes_{S}$ makes $\operatorname{Pic}_{G} S$ an abelian group with identity element $S_{T}$. Here, the $G$-action on a tensor product $X \otimes_{S} Y$ of $T$-modules is diagonal: $(x \otimes y) g=x g \otimes y g$. Following [Kr] $\S 7$ (see also $[\mathrm{DMV}]$ ), we now describe certain maps between $\operatorname{Pic}_{G} S$ and various related groups.

- Most obviously, restricting operators from $T$ to $S$ (forgetting the $G$-operation) yields a group homomorphism $\operatorname{Pic}_{G} S \rightarrow \operatorname{Pic} S$ whose image is clearly contained in the invariants $(\operatorname{Pic} S)^{G}$ of the canonical operation of $G$ on Pic $S$. Thus we have

$$
\sigma: \operatorname{Pic}_{G} S \rightarrow(\operatorname{Pic} S)^{G} .
$$

- Next, restricting the functor $E: \operatorname{proj}-R \rightarrow \operatorname{add}\left(S_{T}\right)$ of $\S 2.1$ to projectives of rank 1 over $R$, we obtain a group monomorphism

$$
\varepsilon: \operatorname{Pic} R \hookrightarrow \operatorname{Pic}_{G} S .
$$


- By general principles (e.g., $[\mathrm{J}])$, the cohomology group $\mathrm{H}^{1}(G, \mathrm{U}(S))$ classifies the $T$-module structures on $S$ which extend the regular action of $S$ on itself: Every derivation (1-cocycle) $d: G \rightarrow \mathrm{U}(S)$ gives rise to a $T$-module structure $S_{d}$ on $S$ via $a \cdot g b=a^{g} d(g) b(a, b \in S, g \in G)$. Conversely, if - is any $T$-module operation on $S$ extending the regular $S$-module structure, then $d(g)=1 \cdot g(g \in$ $G)$ defines a derivation $d \in \operatorname{Der}(G, \mathrm{U}(S))$ so that $(S, \cdot)=S_{d}$. The assignment $d \mapsto S_{d}$ passes down to a map $\delta: \mathrm{H}^{1}(G, \mathrm{U}(S)) \rightarrow \operatorname{Pic}_{G} S$ which fits into an exact sequence

$$
1 \rightarrow \mathrm{H}^{1}(G, \mathrm{U}(S)) \stackrel{\delta}{\longrightarrow} \operatorname{Pic}_{G} S \stackrel{\sigma}{\longrightarrow}(\operatorname{Pic} S)^{G} .
$$

Similarly, there is a map

$$
\rho: \operatorname{Pic}_{G} S \rightarrow \prod_{\mathfrak{M} \in \operatorname{Max} S} \mathrm{H}^{1}\left(G^{T}(\mathfrak{M}),(S / \mathfrak{M})^{*}\right)
$$

obtained by sending the isomorphism class of $P$ to the element whose $\mathfrak{M}$-component is the isomorphism class of $P / P \mathfrak{M}$ as module over $S * G^{T}(\mathfrak{M})$ via restriction from $T=S * G$. Inasmuch as $P / P \mathfrak{M} \cong S / \mathfrak{M}$ as $S / \mathfrak{M}$-modules, the $S * G^{T}(\mathfrak{M})$ module structure on $P / P \mathfrak{M}$ corresponds to an element of $\mathrm{H}^{1}\left(G^{T}(\mathfrak{M}),(S / \mathfrak{M})^{*}\right)$. The foregoing explains most of the following Proposition. For exactness of the second sequence at $\mathrm{Pic}_{G} S$, see $[\mathrm{Kr}]$ or $[\mathrm{DMV}]$.

Proposition. There are exact sequences

$$
1 \rightarrow \mathrm{H}^{1}(G, \mathrm{U}(S)) \stackrel{\delta}{\longrightarrow} \operatorname{Pic}_{G} S \stackrel{\sigma}{\longrightarrow}(\operatorname{Pic} S)^{G}
$$

and

$$
1 \rightarrow \operatorname{Pic} R \stackrel{\varepsilon}{\longrightarrow} \operatorname{Pic}_{G} S \stackrel{\rho}{\longrightarrow} \prod_{\mathfrak{M} \in \operatorname{Max} S} \operatorname{Hom}\left(G^{T}(\mathfrak{M}),(S / \mathfrak{M})^{*}\right) .
$$

The composite $\rho \circ \delta$ is the product $\left\{\rho_{\mathfrak{M}}\right\}$ of the maps

$$
\rho_{\mathfrak{M}}: \mathrm{H}^{1}(G, \mathrm{U}(S)) \rightarrow \mathrm{H}^{1}\left(G^{T}(\mathfrak{M}), \mathrm{U}(S)\right) \rightarrow \operatorname{Hom}\left(G^{T}(\mathfrak{M}),(S / \mathfrak{M})^{*}\right),
$$

the first map being restriction and the second coming from the canonical map $\mathrm{U}(S) \rightarrow(S / \mathfrak{M})^{*}$ and the isomorphism $\mathrm{H}^{1}\left(G^{T}(\mathfrak{M}),(S / \mathfrak{M})^{*}\right) \cong \operatorname{Hom}\left(G^{T}(\mathfrak{M})\right.$, $\left.(S / \mathfrak{M})^{*}\right)$.

\subsection{Picard groups of invariants}

In the special case where $(\operatorname{Pic} S)^{G}$ is trivial, we deduce from Proposition 2.2 that

$$
\operatorname{Pic} R \cong \bigcap_{\mathfrak{M} \in \operatorname{Max} S} \operatorname{Ker} \rho_{\mathfrak{M}} \text {. }
$$

In particular, if $G=G^{T}(\mathfrak{M})$ for some maximal ideal $\mathfrak{M}$ of $S$ such that $\mathrm{U}(S) \rightarrow$ $(S / \mathfrak{M})^{*}$ is an isomorphism, then $\rho_{\mathfrak{M}}$ is an isomorphism as well and so $\operatorname{Pic} R=1$. This applies, for example, to linear actions of $G$ on polynomial algebras, taking $\mathfrak{M}$ the ideal generated by the variables $([\mathrm{K}])$. 


\section{Multiplicative actions}

3.1. We fix the following notations, specializing the setting of $\S 2.1$.
$A$ will denote a (multiplicative) free abelian group of finite rank,
$S \quad$ will be the group algebra $k A$ of $A$ over the field $k$, and
$G$ will be a finite subgroup of $\mathrm{GL}(A)$.

Then $G$ acts as a group of $k$-algebra automorphisms on $S$ by means of the unique extension of the natural $G$-action on $A$. Actions of this type are called multiplicative actions. The notations $T, R$, etc. of $\S 2.1$ remain in effect. Our hypothesis on surjectivity of the trace map is equivalent with the requirement that $|G| \neq 0$ in $k$, which will be assumed throughout.

3.2. Following $[\mathrm{BrL}]$ we put, for each $g \in G$,

$$
I(g)=\left(s-s^{g}: s \in S\right),
$$

the ideal of $S$ that is generated by all elements $s-s^{g}$. Writing $[A, g]$ for the subgroup of $A$ consisting of the elements $[a, g]=a^{-1} a^{g}$, we have the following simple

\section{Lemma.}

(i) For each $\mathfrak{M} \in \operatorname{Max} S, g \in G^{T}(\mathfrak{M})$ if and only if $\mathfrak{M} \supseteq I(g)$.

(ii) $I(g)$ is the kernel of the canonical map $S=k A \rightarrow k A /[A, g]$. In particular, $I(g)$ is semiprime.

Proof. (i) is evident.

(ii). The first assertion is a consequence of the fact that $I(g)$ can clearly also be written as $I(g)=\left(a-a^{g}: a \in A\right)=([a, g]-1: a \in A)$. So $S / I(g)$ is isomorphic with the group algebra $k A /[A, g]$ and, in order to prove semiprimeness, we must show that the order of the torsion subgroup of $A /[A, g]$ is nonzero in $k$ (cf. $[\mathrm{P}]$, Theorems 4.2.12/13). But, letting $\gamma$ denote the endomorphism $a \mapsto \prod_{x \in\langle g\rangle} a^{x}$ of $A$, the torsion subgroup of $A /[A, g]$ is $(\operatorname{Ker} \gamma) /[A, g] \cong \mathrm{H}^{1}(\langle g\rangle, A)$, a finite abelian group whose exponent divides the order of $g$. Our assertion now follows from the fact that $|G| \neq 0$ in $k$.

3.3. Theorem. Pic $R \cong \bigcap_{C} \operatorname{Ker}\left(\operatorname{Res}_{C}^{G}: \mathrm{H}^{1}(G, A) \rightarrow \mathrm{H}^{1}(C, A)\right)$, where $C$ runs over the cyclic subgroups of $G$.

Proof. First, $S$ is a UFD, so Pic $S=1$ and $\S 2.3$ yields

$$
\operatorname{Pic} R \cong \bigcap_{\mathfrak{M} \in \operatorname{Max} S} \operatorname{Ker} \rho_{\mathfrak{M}}
$$


Next, $S$ has only "trivial" units, $\mathrm{U}(S)=k^{*} \times A$. Hence,

$$
\mathrm{H}^{1}(G, \mathrm{U}(S))=\mathrm{H}^{1}(G, A) \times \operatorname{Hom}\left(G, k^{*}\right) .
$$

Moreover, for the augmentation ideal $\omega S=(a-1: a \in A)$ of $S$, the map $\rho_{\omega S}: \mathrm{H}^{1}(G, \mathrm{U}(S)) \rightarrow \operatorname{Hom}\left(G^{T}(\omega S),(S / \omega S)^{*}\right)=\operatorname{Hom}\left(G, k^{*}\right)$ is trivial on $\mathrm{H}^{1}(G, A)$ and the identity on $\operatorname{Hom}\left(G, k^{*}\right)$. Therefore,

$$
\operatorname{Pic} R \cong \bigcap_{\mathfrak{M} \in \operatorname{Max} S} \operatorname{Ker} r_{\mathfrak{M}}
$$

where $r_{\mathfrak{M}}$ denotes the restriction of $\rho_{\mathfrak{M}}$ to $\mathrm{H}^{1}(G, A)$. Writing $\mathrm{cl}(d)$ for the class of the derivation $d \in \operatorname{Der}(G, A)$ in $\mathrm{H}^{1}(G, A)$, we have

$$
\begin{aligned}
\operatorname{cl}(d) \in \bigcap_{\mathfrak{M} \in \operatorname{Max} S} \operatorname{Ker} r_{\mathfrak{M}} & \Longleftrightarrow \forall \mathfrak{M} \in \operatorname{Max} S \forall g \in G^{T}(\mathfrak{M}): \quad d(g)-1 \in \mathfrak{M} \\
& \Longleftrightarrow \forall g \in G: \quad d(g)-1 \in \bigcap_{\substack{\mathfrak{M} \in \operatorname{Max} S \\
\mathfrak{M} \supseteq I(g)}} \mathfrak{M} \\
& \Longleftrightarrow \forall g \in G: \quad d(g) \in[A, g]
\end{aligned}
$$

The second and the third of the above equivalences follow from Lemma 3.2(i) and (ii), respectively, and the last statement asserts that, for all $g \in G$, the restriction of $\operatorname{cl}(d)$ to $\langle g\rangle$ is trivial in $\mathrm{H}^{1}(\langle g\rangle, A)$, which completes the proof of the theorem. $\square$

\subsection{Alternative description}

The cohomology group $\mathrm{H}^{1}(G, A)$ can be written as follows:

$$
\mathrm{H}^{1}(G, A) \cong \bar{A}^{G} / \overline{A^{G}}
$$

where ${ }^{-}: A \rightarrow \bar{A}=A / A^{n}$ is the canonical map and $n=|G|$ is the order of $G$ (or any multiple). The above isomorphism follows from the cohomology sequence for $1 \rightarrow A \stackrel{(.)^{n}}{\longrightarrow} A \stackrel{-}{\longrightarrow} \rightarrow 1$ which starts with

$$
1 \longrightarrow A^{G} \longrightarrow A^{G} \stackrel{\longrightarrow}{\longrightarrow} \bar{A}^{G} \longrightarrow \mathrm{H}^{1}(G, A) \stackrel{(.)^{n}=0}{\longrightarrow} \mathrm{H}^{1}(G, A)
$$

(e.g., [B], §15.5). Explicitly, the cohomology class that is associated with the element $a A^{n} \in \bar{A}^{G}$ for $a \in A$ is the class of the derivation

$$
d_{a}: G \rightarrow A, \quad g \mapsto \sqrt[n]{[a, g]} .
$$


In these terms, the restriction map $\operatorname{Res}_{C}^{G}: \mathrm{H}^{1}(G, A) \rightarrow \mathrm{H}^{1}(C, A)$ is simply the map

$$
\bar{A}^{G} / \overline{A^{G}} \longrightarrow \bar{A}^{C} / \overline{A^{C}}
$$

that is afforded by the inclusions $\bar{A}^{G} \subseteq \bar{A}^{C}$ and $\overline{A^{G}} \subseteq \overline{A^{C}}$. This leads to the following easily programmeable description of Pic $R$ :

$$
\operatorname{Pic} R \cong\left(\bigcap_{\substack{C \leq G \\ C \text { cyclic }}} \overline{A^{C}}\right) / \overline{A^{G}} .
$$

\subsection{Relationship with the class group}

For general reasons (e.g., [F], Cor. 18.3), Pic $R$ embeds into the class group $\mathrm{Cl} R$ of $R$. The latter group has been determined in $[\mathrm{L}]$ :

$$
\mathrm{Cl} R \cong \operatorname{Hom}\left(G / N, k^{*}\right) \oplus \mathrm{H}^{1}\left(G / D, A^{D}\right),
$$

where $N$ denotes the subgroup of $G$ that is generated by all reflections in $G$ and $D$ the subgroup generated by the reflections that are diagonalizable over $\mathbb{Z}$. An explicit embedding Pic $R \hookrightarrow \mathrm{Cl} R$ can be realized as follows. The term $\mathrm{H}^{1}\left(G / D, A^{D}\right)$ is canonically isomorphic with $\bigcap_{H} \operatorname{Ker}\left(\operatorname{Res}_{H}^{G}: \mathrm{H}^{1}(G, A) \rightarrow \mathrm{H}^{1}(H, A)\right)$, where $H$ runs over the subgroups of $G$ that are generated by a $\mathbb{Z}$-diagonalizable reflection ([L], proof of Theorem 2.5). As all these occur among the subgroups $C$ in Theorem 3.3 , there is an obvious inclusion (strict, in general)

$$
\operatorname{Pic} R \subseteq \mathrm{H}^{1}\left(G / D, A^{D}\right) \text {. }
$$

\subsection{The Cartan map}

For any integrally closed commutative Noetherian domain $\mathcal{R}$, the embedding $\mathrm{Pic} \mathcal{R} \hookrightarrow \mathrm{Cl} \mathcal{R}$ that was referred to in $\S 3.5$ is part of a commutative diagram involving the Cartan map $c_{\mathcal{R}}: K_{0}(\mathcal{R}) \rightarrow G_{0}(\mathcal{R})$ :

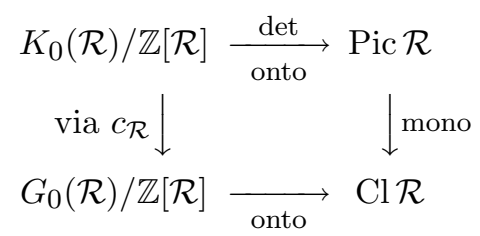

The map det is described in [Ba], p. 466 and the map $G_{0}(\mathcal{R}) / \mathbb{Z}[\mathcal{R}] \rightarrow \mathrm{Cl} \mathcal{R}$ is as in $[\mathrm{Bou}]$, p. 69. Commutativity of the diagram is a consequence of [Bou], Exercise 12(b), p. 108.

In view of this diagram, the examples of multiplicative invariants $R$ with nontrivial Pic $R$ which we will exhibit in $\S 3.8$ also have the property that $\operatorname{Im} c_{R}$ is strictly larger than $\mathbb{Z}[R]$. This is in contrast with the case of linear invariants $R$, where one always has $\operatorname{Im} c_{R}=\mathbb{Z}[R]$. (See the Appendix.) 


\subsection{Torsion in $K_{0}$ of some polycyclic group algebras}

Note that, in the setting of $\S 3.1$,

$T \cong k \Gamma$, the group algebra of the semidirect product $\Gamma=A \rtimes G$ over $k$.

Thus $\Gamma$ is a polycyclic-by-finite group. We will use our observations about the Cartan map $c_{R}$ in $\S 3.6$ to show:

Proposition. Pic $R$ is a subquotient of the torsion subgroup of $K_{0}(T)$.

Proof. Consider the following commutative diagram:

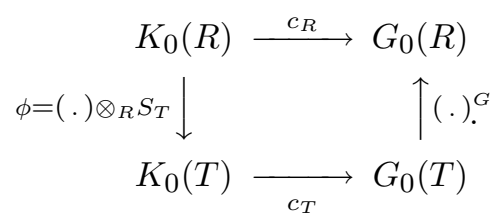

That this diagram is indeed commutative is a consequence of our remarks about the functors $E$ and $F$ in $\S 2.1$. Our assumption on $|G|$ implies that $T$ is regular, and so $c_{T}$ is an isomorphism. (By [BrL], Cor. 1.3, one further knows that the $G$ fixed point map $(.)^{G}: G_{0}(T) \rightarrow G_{0}(R)$ is surjective, but this will not be essential here.) Our goal is to establish the following

Claim. $\operatorname{Im} \phi=\mathbb{Z}\left[S_{T}\right] \oplus \mathcal{T}$ for some finite subgroup $\mathcal{T}$ of $K_{0}(T)$.

Granting this for now, we conclude that the image of the map $\overline{c_{R}}: K_{0}(R) / \mathbb{Z}[R] \rightarrow$ $G_{0}(R) / \mathbb{Z}[R]$ that was considered in $\S 3.6$ is identical with the image of $\mathcal{T}$ in $G_{0}(R) / \mathbb{Z}[R]$. Thus, $\operatorname{Im} \overline{c_{R}}$ is a subquotient of the torsion subgroup of $K_{0}(T)$. Moreover, from the diagram in $\S 3.6$ we infer that $\operatorname{Pic} R$ is a subquotient of $\operatorname{Im} \overline{c_{R}}$, whence the proposition.

To prove the claim, note that $K_{0}(S)=\mathbb{Z} \cdot[S]$. Hence $K_{0}(T)=\mathbb{Z}\left[S_{T}\right] \oplus \operatorname{Ker} \operatorname{Res}_{S}^{T}$ and $\operatorname{Im} \phi=\mathbb{Z}\left[S_{T}\right] \oplus \mathcal{F}$, where $\mathcal{T}=\operatorname{Im} \phi \cap \operatorname{Ker}_{\operatorname{Res}_{S}^{T}}^{T}$. To prove that $\mathcal{T}$ is finite, we consider the canonical map $\sim: T=k \Gamma \rightarrow \widetilde{T}=k[\Gamma / B]$ for a suitable normal subgroup $B$ of $\Gamma$. Specifically, following [BrL2], we can choose $B$ to be a subgroup of $A$ such that the quotient $\widetilde{\Gamma}=\Gamma / B \cong(A / B) \rtimes G$ has the following properties:

(1) $\widetilde{\Gamma}$ is finite and its order divides a power of $|G|$, and

(2) the map $K_{0}(\widetilde{\sim}): K_{0}(T) \rightarrow K_{0}(\widetilde{T})$ has a finite kernel.

We briefly indicate the relevant results from [BrL2], using the notation and terminology of that article: The construction of $B$ is given in [BrL2] $\S 1.7$ (where $\widetilde{\Gamma}$ is denoted $G)$. Property $(2)$ is a consequence of fullness of the functor $\mathcal{F}(\widetilde{\sim})$ : $\mathcal{F}(\Gamma) \rightarrow \mathcal{F}(\widetilde{\Gamma})$ between the Frobenius categories of finite subgroups of $\Gamma$ and $\widetilde{\Gamma}$. Indeed, putting $\mathcal{X}=\{\widetilde{X} \mid X \in \mathcal{F}(\Gamma)\}$ as in [BrL2] $\S 1.6$, we have a commutative diagram 


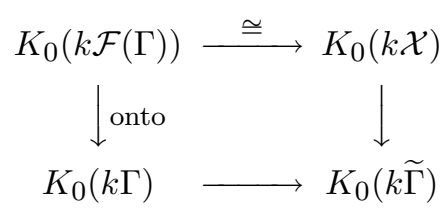

with all maps the obvious induction homomorphisms. The fact that the map on top is an isomorphism is [BrL2], Lemma 1.6(i). The map on the left is surjective, by Moody's induction theorem [M], and the map on the right has a finite kernel, by [BrL2], Proposition 1.8. Consequently, the bottom map has a finite kernel as well, whence (2).

Thus it suffices to show that $K_{0}(\stackrel{\sim}{.})$ annihilates $\mathcal{T}$. But $\widetilde{T}=k \widetilde{\Gamma} \cong \widetilde{S} * G$, and so we have an analogous map $\widetilde{\phi}=(.) \otimes_{\widetilde{R}} \widetilde{T}: K_{0}(\widetilde{R}) \rightarrow K_{0}(\widetilde{T})$, where $\widetilde{R}=\widetilde{S}^{G}$.

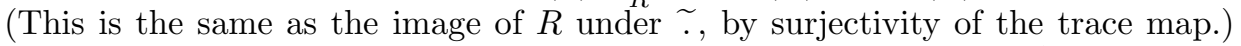
Furthermore, $K_{0}(\widetilde{r})$ sends $\mathcal{T}=\operatorname{Im} \phi \cap \operatorname{Ker} \operatorname{Res}_{S}^{T}$ to $\operatorname{Im} \widetilde{\phi} \cap \operatorname{Ker} \operatorname{Res} \widetilde{\widetilde{T}}$, and so we are reduced to showing that the latter intersection is trivial. However, $\operatorname{Re} \frac{\widetilde{T}}{\widetilde{S}} \circ \widetilde{\phi}$ is identical with the induction map $\operatorname{Ind}_{\widetilde{R}} \widetilde{S}: K_{0}(\widetilde{R}) \rightarrow K_{0}(\widetilde{S})$, and this map is injective, since $\widetilde{R}$ and $\widetilde{S}$ are commutative Artinian rings with $\widetilde{R} \subseteq \widetilde{S}$. This completes the proof of the proposition.

\subsection{Computations in dimension $\leq 4$}

A search of the crystallographic groups library of $G A P$ produced the following results.

3.8.1. Dimension 2. All multiplicative invariants $R$ for $\operatorname{rk} A=2$ have trivial Picard group. This is easy to see directly: Since $\mathrm{SL}_{2}(\mathbb{Z})$ acts fixed point freely on $\mathbb{Z}^{2}$, we have $A^{C}=0$ for every nontrivial cyclic subgroup $C \subseteq \mathrm{SL}(A)$. Consequently, $\operatorname{Pic} R=1$ if $G$ intersects $\operatorname{SL}(A)$ nontrivially. In the opposite case, $G$ is cyclic, having order at most 2 , and so Pic $R=1$ again.

3.8.2. Dimension 3. Among the 73 conjugacy classes of finite subgroups of $\mathrm{GL}_{3}(\mathbb{Z})$, it turns out, 2 lead to an invariant algebra $R$ with nontrivial Picard group. These classes are represented by the groups $G_{1}=\langle x, y\rangle \cong C_{2} \times C_{2}$ and $G_{2}=\langle x, y, z\rangle \cong$ $A_{4}$, where $x=\left(\begin{array}{lll}0 & -1 & 1 \\ 0 & -1 & 0 \\ 1 & -1 & 0\end{array}\right), y=\left(\begin{array}{ccc}0 & 1 & -1 \\ 1 & 0 & -1 \\ 0 & 0 & -1\end{array}\right)$, and $z=\left(\begin{array}{ccc}0 & 0 & 1 \\ 1 & 0 & 0 \\ 0 & 1 & 0\end{array}\right)$. In both cases, Pic $R$ has order 2. Note that $G_{1}$ is a normal subgroup of $G_{2}$. An explicit isomorphism $\operatorname{Pic} S^{G_{2}} \stackrel{\cong}{\longrightarrow} \operatorname{Pic} S^{G_{1}}$ is afforded by the restriction map $\mathrm{H}^{1}\left(G_{2}, A\right) \stackrel{\cong}{\longrightarrow}$ $\mathrm{H}^{1}\left(G_{1}, A\right)^{G_{2}}$ (note that $\left.A^{G_{1}}=0\right)$. We remark that $\mathrm{Cl} S^{G_{1}}=\mathbb{Z} /(2) \oplus \mathbb{Z} /(2) \oplus \mathbb{Z} /(4)$ and $\mathrm{Cl} S^{G_{2}}=\mathbb{Z} /(3) \oplus \mathbb{Z} /(4)$.

3.8.3. Dimension 4. The 710 conjugacy classes of finite subgroups of $\mathrm{GL}_{4}(\mathbb{Z})$ altogether include 9 whose invariant algebra has a nontrivial Picard group, of 
order 2 in all cases. The above two rank 3 groups $G$ directly account for 4 of these: $\left(\begin{array}{ll}G & \\ & 1\end{array}\right)$ has invariant algebra $R\left[X^{ \pm 1}\right]$ and $\left(\begin{array}{ll}G & \\ & \pm 1\end{array}\right)$ leads to $R[X]$, both with the same Picard group (and class group) as $R$ (cf. [Ba], Props. III.7.17 and III.7.19).

\section{Appendix: The Cartan map for linear actions}

The purpose of this short Appendix is to substantiate the claim, made in $\S 3.6$, that for invariants $R$ of linear actions, the image of the Cartan map $c_{R}$ is generated by the classes of the free $R$-modules. We will use the following notations:
$V \quad$ will denote a finite-dimensional vector space over the field $k$,
$S \quad$ will be the symmetric algebra $S(V)$, and
$G \quad$ will be a finite subgroup of $\operatorname{GL}(V)$ with $|G| \neq 0$ in $k$.

We consider the $G$-action by $k$-algebra automorphisms on $S$ obtained by extending the natural action on $V$. The remaining notations $R, T$, etc. are as in $\S 2.1$.

Proposition. $\operatorname{Im} c_{R}=\mathbb{Z}[R]$.

Proof. The proof is a greatly simplified version of the proof of Proposition 3.7 whose notation we employ here. It suffices to show that $\operatorname{Im} \phi=\mathbb{Z}\left[S_{T}\right]$ and, exactly as in the proof of Proposition 3.7, one certainly has $\operatorname{Im} \phi=\mathbb{Z}\left[S_{T}\right] \oplus \mathcal{T}$, where $\mathcal{T}=\operatorname{Im} \phi \cap \operatorname{Ker} \operatorname{Res}_{S}^{T}$. To prove that $\mathcal{T}=0$, consider the map $\tilde{.}: T=S * G \rightarrow \widetilde{T}=$ $k G$ which sends $V$ to 0 . By Quillen's induction theorem ([Q], Theorem 7), the corresponding map $K_{0}(\sim)$ is an isomorphism. On the other hand, a trivial variant of the argument used in the proof of Proposition 3.7, with $\widetilde{R}=\widetilde{S}=k$, shows that $K_{0}(\stackrel{\sim}{.})$ annihilates $\mathcal{T}$, whence $\mathcal{T}=0$.

\section{References}

[B] A. Babakhanian, Cohomological Methods in Group Theory, Marcel Dekker, New York 1972.

[Ba] H. Bass, Algebraic K-theory, Benjamin, New York 1968.

[Bou] N. Bourbaki, Algèbre commutative, chap. 7, Hermann, Paris 1965.

[BrL] K.A. Brown and M. Lorenz, Grothendieck groups of invariant rings and of group rings, J. Algebra 166 (1994), 423-454.

[BrL2] K.A. Brown and M. Lorenz, Colimits of functors and Grothendieck groups of infinite group algebras, Contemp. Math. 130 (1992), 89-109.

[F] R.M. Fossum, The Divisor Class Group of a Krull Domain, Springer-Verlag, Berlin 1973.

[H] T.J. Hodges, Equivariant $K$-theory for Noetherian rings, J. London Math. Soc. 39 (1989), 414-426. 
[Ho] M. Holland, $K$-theory of endomorphism rings and of rings of invariants, J. Algebra 191 (1997), 668-685.

[J] N. Jacobson, Forms of algebras. In: Some Recent Advances in Basic Sciences, Academic Press, New York 1966, pp. 41-71. See also Collected Mathematical Papers 3, Birkhäuser, Boston 1989, pp. 399-429.

[K] M.-C. Kang, Picard groups of some rings of invariants, J. Algebra 58 (1979), 455-461.

[Kr] H. Kraft, $G$-vector bundles and the linearization problem. In: Canadian Mathematical Soc. Conference Proceedings Vol. 10, Amer. Math. Soc., Providence, R.I. 1989, pp. $111-123$.

[DMV] H. Kraft, P. Slodowy, and T.A. Springer (eds.), Algebraic Transformation Groups and Invariant Theory, DMV Seminar, Band 13, Birkhäuser Verlag, Basel 1989.

[KM] P.H. Kropholler and B. Moselle, A family of crystallographic groups with 2-torsion in $K_{0}$ of the rational group algebra, Proc. Edin. Math. Soc. 34 (1991), 325-331.

[L] M. Lorenz, Class groups of multiplicative invariants, J. Algebra 177 (1995), 242-254.

[M] J.A. Moody, A Brauer induction theorem for $G_{0}$ of certain infinite groups, J. Algebra 122 (1989), 1-14.

[P] D.S. Passman, The Algebraic Structure of Group Rings, Wiley\& Sons, New York 1977.

[Q] D. Quillen, Higher algebraic K-theory I. In: Lect. Notes in Math. No. 341 (H. Bass, ed.), Springer-Verlag, Berlin 1973, pp. 85-147.

[Sch] M. Schönert et. al., GAP: Groups, Algorithms and Programming (version 3.4), Lehrstuhl D für Mathematik, RWTH Aachen, Germany, 1994.

\section{Martin Lorenz}

Department of Mathematics

Temple University

Philadelphia, PA 19122-6094

e-mail: lorenz@math.temple.edu

(Received: April 25, 1996) 